\title{
Increased expression of high-mobility group nucleosomal-binding domain 2 protein in various tumor cell lines
}

\author{
QIAN LI* , JIAO CHEN ${ }^{*}$, XIAOYING LI, BOMIAO CUI, YAPING FAN, NING GENG, \\ QIANMING CHEN, PING ZHANG and YUN FENG

\begin{abstract}
State Key Laboratory of Oral Diseases, West China School of Stomatology, Sichuan University, Chengdu, Sichuan 610041, P.R. China
\end{abstract}

Received April 12, 2017; Accepted December 14, 2017

DOI: $10.3892 / \mathrm{ol} .2018 .7898$

\begin{abstract}
High mobility group nucleosomal-binding domain 2 (HMGN2) is an abundant non-histone nuclear protein of vertebrates and invertebrates. The aim of the present study was to characterize the endogenous expression of HMGN2 in various types of tumor cell. Western blotting was performed to analyze HMGN2 expression in the following tumor cell lines: H1975, HSC-4, MDA-MB-468, MDA-MB-231, SCC-25 and THP-1. Periodontal ligament cells (PDLCs) were included as a noncancerous control. HMGN2 was detected in human oral squamous cell carcinoma tissues by immunohistochemical analysis. The results demonstrated that the expression of HMGN2 was increased in the majority of tumor cell lines, particularly MDA-MB-468 and THP-1 cells, compared with PDLCs. The expression of HMGN2 in oral squamous cell carcinoma tissue was significantly increased compared with the expression in normal tissue. Furthermore, the expression of HMGN2 in metastatic oral squamous cell carcinoma tissues was increased compared with that in its non-metastatic counterpart. These results indicated that HMGN2 may serve an important function in the growth and metastasis of tumor cells.
\end{abstract}

Correspondence to: Dr Ping Zhang or Dr Yun Feng, State Key Laboratory of Oral Diseases, West China School of Stomatology, Sichuan University, 14 Renmin South Road Section 3, Chengdu, Sichuan 610041, P.R. China

E-mail: pingzhang68@hotmail.com

E-mail: 953463551@qq.com

${ }^{*}$ Contributed equally

Abbreviations: HMGN2, high mobility group nucleosomal-binding domain 2; OSCC, oral squamous carcinoma; PDLCs, periodontal ligament cells; shRNAs, small hairpin RNAs

Key words: high mobility group nucleosomal-binding domain 2, tumor cells, oral squamous cell carcinoma

\section{Introduction}

The high mobility group (HMG) proteins were originally isolated from the nuclei of mammalian cells and named according to their electrophoretic mobility (1). HMGs were later subdivided into three families: HMG-AT-hook family (HMGA), the HMG-box family (HMGB) and the HMG-nucleosome binding family (HMGN), each comprising several protein members (2). The HMGN family includes five chromatin architectural proteins, which are present in higher vertebrates (3). A number of previous studies have demonstrated that HMGNs are transcriptional co-regulators that serve important functions in DNA repair and cancer progression (3-6). HMGN2 is the most conserved member of the HMGN family, involved in unfolding higher-order chromatin structures and facilitating the transcriptional activation of various mammalian genes $(7,8)$. However, the biological and pathological functions of HMGN2 are not well understood. In the present study, HMGN2 expression was evaluated in tumor cells and human oral squamous cell carcinoma (OSCC) tissues. The results demonstrated that the expression of HMGN2 was significantly increased in the majority of tumor cell lines compared with periodontal ligament cells (PDLCs). Furthermore, HMGN2 expression was increased in human OSCC tissues compared with normal oral tissues. In metastatic OSCC tissues, HMGN2 expression was increased, compared with non-metastatic OSCC tissues.

\section{Materials and methods}

Ethical approval. The Committee for Ethical Approval and the State Key Laboratory of Oral Diseases of the West China School of Stomatology, Sichuan University (Chengdu, China) approved the present study (reference no. WCHSIRB-ST-2015-147). Protocols regarding the use and manipulation of PDL tissues were approved by the Institutional Review Board of West China Hospital of Stomatology, Sichuan University (Institutional Review Board reference number: WCHSIRB-D-2013-039). All PDLCs and OSSC tissues donors have provided written informed consent.

Isolation and culture of periodontal ligament cells (PDLCS). PDL tissues were isolated from the extracted premolar teeth 
of three healthy donors (two females and one male aged $14 \pm 3$ years old) who received orthodontic treatment at the West China Hospital of Stomatology of Sichuan University from July to September in 2016. Teeth with caries, periodontitis and periapical periodontitis were excluded. The extracted teeth were rinsed with Dulbecco's Modified Eagle Medium (HyClone; GE Healthcare, Chicago, IL, USA) supplemented with $100 \mathrm{U} / \mathrm{ml}$ penicillin and $100 \mu \mathrm{g} / \mathrm{ml}$ streptomycin. The remaining procedures for PDLC extraction were performed as previously described by Arnold and Baram (9).

Western blotting and densitometry. The following human cell lines were kindly donated by Dr Qianming Chen (West China Hospital of Stomatology of Sichuan University), who purchased them from The American Type Culture Collection (Manassas, VA, USA) and cultured them at the State Key Laboratory of Oral Diseases; these included the lung adenocarcinoma cell line, H1975; oral squamous carcinoma cell line, HSC-4; triple negative breast cancer cell line, MDA-MB-468; tripe negative breast cancer cell line, MDA-MB-231; oral squamous carcinoma cell line, SCC-25, and leukemia cell line, THP-1. The H1975, HSC-4, MDA-MB-2x3, MDA-MB-468 and THP-1 cell lines were cultured in RPMI 1640, the SCC-25 cell line was cultured in DEM/F-12 (Hyclone; GE Healthcare) and the PDLC cell line was cultured in $\alpha$-MEM medium (all media were purchased from Hyclone; GE Healthcare). All cell lines were supplemented with $10 \%$ fetal calf serum (Hyclone; GE Healthcare), $100 \mathrm{mg} / \mathrm{ml}$ streptomycin and $100 \mathrm{IU} / \mathrm{ml}$ penicillin (both Gibco; Thermo Fisher Scientific Inc.). All tumor cell lines and PDLCs were cultured in a humidified incubator at $37^{\circ} \mathrm{C}$ with $5 \% \mathrm{CO}_{2}$, and all experiments were conducted while cells were in the logarithmic growth phase. Total protein was extracted with a total protein extraction kit (Jiangsu, Keygen Biotech Co., Ltd., Jiang Su, China), according to the manufacturer's protocol. Total protein concentration was determined by BCA assay (Thermo Fisher Scientific Inc., Waltham, MA, USA), and equal amounts of protein $(20 \mu \mathrm{g})$ from each sample were separated by SDS-PAGE (12\% gel) according to the molecular weights of the tested proteins. Proteins were then transferred onto Immun-Blot polyvinylidenedifluoride membranes (Invitrogen; Thermo Fisher Scientific, Inc.) and blocked with 5\% skimmed milk at room temperature for $1 \mathrm{~h}$. Anti-HMGN2 and anti- $\beta$-actin primary antibodies (both diluted 1:1,000; catalog nos. ab199679 and ab8226; Abcam, Cambridge, UK) were incubated with the membranes at $4^{\circ} \mathrm{C}$ overnight. Bound antibodies were detected by incubation with corresponding horseradish peroxidase-conjugated secondary antibodies (dilution, 1:2,000; catalog no. ZB-2301; OriGene Technologies, Inc., Beijing, China) at room temperature for $2 \mathrm{~h}$. Following three washes in tris-buffered saline with Tween 20, immunoreactive bands were detected using the Easy ECL Western Blot kit (TransGen Biotech, Inc., Beijing, China) and captured using the Chemidoc XRS system (Bio-Rad Laboratories, Inc., Hercules, CA, USA). Quality One software version 4.62 (Bio-Rad Laboratories, Inc.) was used to quantify relative protein level in different cell lines; these levels were normalized to the concentration of $\beta$-actin. Experiments for each cell line were performed in triplicate.
Immunohistochemical analysis. Sections of human OSCC and normal oral tissues, including 2 cases of tongue, 2 of palate, 1 of gingiva non-metastatic OSCC and 1 of gingival, oropharynx, tongue, cheek and floor of mouth metastatic OSCC respectively, in addition to 1 case of normal tissue serving as a control. These tissues were evaluated for HMGN2 expression by immunohistochemical analysis. A total of 11 specimens were selected from the Department of Pathology of West China Hospital of Stomatology (Chengdu, China) between March and September in 2016, where 5 metastatic OSCC specimens, 5 non-metastatic OSCC specimens and 1 non-neoplastic oral tissue specimen were included. The clinicopathological information of patients providing theses specimens are provided in Table I. Tissues were fixed with $4 \%$ paraformaldehyde for $24 \mathrm{~h}$ at $4^{\circ} \mathrm{C}$ and embedded in paraffin. Tissues sections of 4- $\mu \mathrm{m}$ thickness were subsequently deparaffinized with xylene and rehydrated in a graded series of ethanol $(95,90$, 80 and $75 \%$ ). Endogenous peroxidase activity was quenched with $0.3 \% \mathrm{H}_{2} \mathrm{O}_{2}$ for $10 \mathrm{~min}$. Heat-induced antigen retrieval was carried out in $0.5 \mathrm{~mol} / 1$ of ethylenediaminetetraacetic acid (pH 7.0) in a pressure cooker for $5 \mathrm{~min}$. Nonspecific binding was blocked using 10\% normal goat serum (Nanjing KeyGen Biotech Co., Ltd., Nanjing, China) at $37^{\circ} \mathrm{C}$ for $30 \mathrm{~min}$ prior to incubation with the primary antibody (dilution, 1:50; catalog no. ab199679; Abcam) in PBS overnight. According to the manufacture's protocol, slides were rewarmed at $37^{\circ} \mathrm{C}$ for $1 \mathrm{~h}$ and then stained with anti-rabbit biotinylated second antibody at $37^{\circ} \mathrm{C}$ for another $1 \mathrm{~h}$, followed by an incubation with a streptavidin-horseradish peroxidase system for $30 \mathrm{~min}$ at $37^{\circ} \mathrm{C}$ (catalog no. KGSP03-KGSP04; Nanjing KeyGen Biotech Co., Ltd.). The antigen-antibody reaction was visualized using 3,3'-diaminobenzidine, with positive cells staining brown. The sections were subsequently counterstained with $0.2 \%$ hematoxylin for $30 \mathrm{sec}$ at room temperature, then dehydrated in a series of ethanol washes $(80,90,95$ and $100 \%$ respectively), dried, and mounted onto coverslips. Images of representative areas of each sample were captured under light microscopy at $\mathrm{x} 40$ and $\mathrm{x} 400$ magnification (Nikon Corporation, Tokyo, Japan).

Total staining score for human OSCC and normal oral tissues was calculated as the product of the intensity score of HMGN2 and the percentage of HMGN2-positive cells. As a result, the higher the total staining score, the stronger the expression of HMGN2. Specific scoring was applied according to the following criteria: i) The intensity scores of HMGN2 were as follows: Strong (4); moderate (3); mild (2); and negative (1); ii) A section was considered positive based on brown staining in the cell nucleus, and the mean percentage of positive cells in 5 fields of vision at $\times 400$ magnification was scored as follows: 0 point, $<5 \%$; 1 point, $5-25 \%$; 2 points, $26-50 \%$; 3 points, $51-75 \%$; and 4 points, $>75 \%$; iii) HMGN2 expression was acquired by multiplying the intensity score by the percentage of positive cells (points) to produce a total score: $0-4$, negative; 4-8, mild; $8-12$, moderate; $12-16$, marked. D) Experiments were performed in triplicate and two pathologists evaluated total score independently in each experiment.

Statistical analysis. SPSS software v20.0 (IBM Corp., Armonk, NY, USA) was adopted for data analysis. According to data type, a one-way analysis of variance and then Dunnett- $t$ 
Table I. Clinicopathological information of patients who provided tissue for immunohistochemistry.

\begin{tabular}{llcl}
\hline Tissue type & Sex & Age, years & \multicolumn{1}{c}{ Pathological diagnosis } \\
\hline Non-neoplastic & Male & 25 & Tongue tissue (normal) \\
& Male & 48 & SCC (Phase I-II) \\
& Female & 44 & SCC (Phase II) \\
Non-metastatic OSCC & Male & 69 & SCC (Phase II), with eosinophilic infiltration \\
& Male & 50 & SCC (Phase I) \\
& Male & 62 & SCC (Phase I-II), with salivary gland infiltration \\
& Male & 61 & SCC (Phase II), with metastasis to superior deep cervical lymphatic nodes \\
& Male & 50 & SCC (Phase II-III), with metastasis to superior deep cervical lymphatic nodes \\
Metastatic OSCC & Male & 63 & SCC (Phase I), with metastasis to submental submandibular lymphatic nodes \\
& & & and superior, middle and inferior deep cervical lymphatic nodes \\
& Male & 69 & SCC (Phase I), with metastasis to superior deep cervical lymphatic nodes \\
& Male & 57 & SCC (Phase I and II), with muscle layer and salivary gland infiltration; with \\
& & & metastasis to superior deep cervical lymphatic nodes
\end{tabular}

OSCC, oral squamous cell carcinoma.

test were used to identify differences in HMGN2 expression among various cell lines. In addition, the Kruskal-Wallis test followed by Student-Newman-Keuls post hoc test were applied to evaluate significance among three groups of tissues. $\mathrm{P}<0.05$ was considered to indicate a statistically significant difference.

\section{Results}

HMGN2 expression is increased in tumor cell lines. The protein expression level of HMGN2 in tumor cell lines was analyzed using western blotting. H1975, HSC-4, MDA-MB-468, MDA-MB-231, SCC-25 and THP-1 cells were selected for analysis of HMGN2 expression. PDLCs were used as noncancerous control cells. The HMGN2 expression levels in these cell lines are presented in Fig. 1.

All tumor cells with the exception of the H1975 cell line demonstrated increased expression of HMGN2 compared with PDLCs. In particular, MDA-MB-468 cells and THP-1 cells exhibited marked HMGN2 expression levels (Fig. 1A and B). Compared with PDLCs, the expression of HMGN2 was $>10$-fold greater in MDA-MB-468 cells and >5-fold greater in THP-1 cells (Fig. 1B). The expression level of HMGN2 in HSC-4, MDA-MB-231 and SCC-25 cells were also increased compared with PDLCs (Fig. 1B).

HMGN2 is overexpressed in human OSCC tissues. HMGN2 expression in human OSCC and control tissues was evaluated using immunohistochemistry. A total of 5 metastatic and 5 non-metastatic OSCC tissues, and 1 non-neoplastic oral tissue were included in this analysis. Representative areas of each sample were captured, and the number of positive (i.e., stained) cells, as well as the staining intensity, were determined. As presented in Fig. 2, positive staining for HMGN2 was primarily nuclear-localized and populated basal, tumor and inflammatory cells. These results supported the hypothesis that HMGN2 is involved in cell proliferation, inflammation and tumorigenesis. In noncancerous control specimens, low or absent anti-HMGN2
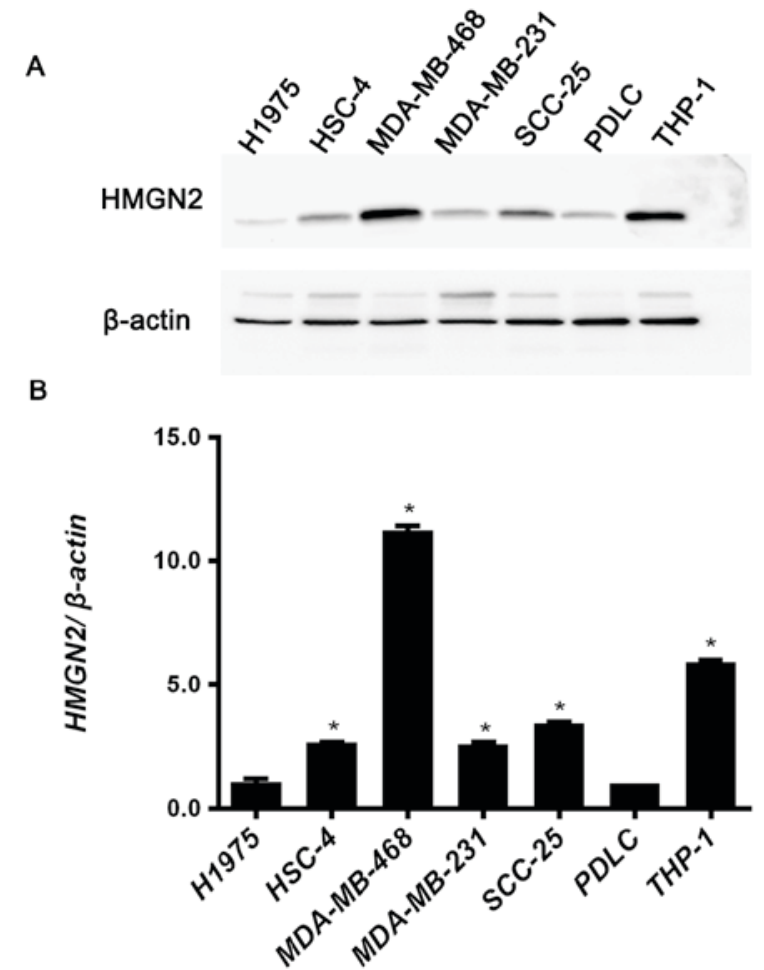

Figure 1. HMGN2 is expressed at an increased level in tumor cell lines. H1975, HSC-4, MDA-MB-468, MDA-MB-231, SCC-25 and THP-1 tumor cell lines were investigated, compared with PDLCs noncancerous control. (A) HMGN2 expression was detected via western blotting. (B) Western blotting was quantified; the error bars are representative of the mean \pm standard deviation of three independent experiments. ${ }^{*} \mathrm{P}<0.05$ vs. PDLCs. HMGN2, high mobility group nucleosomal-binding domain 2; PDLCs, periodontal ligament cells.

immunoreactivity was observed in basal cells. Nevertheless, the overwhelming majority of squamous and inflammatory cells exhibited markedly positive staining for anti-HMGN2, particularly in metastatic OSCC tissues. In 5 non-metastatic OSCC tissues, 4 cases $(80 \%)$ exhibited moderate nuclear 

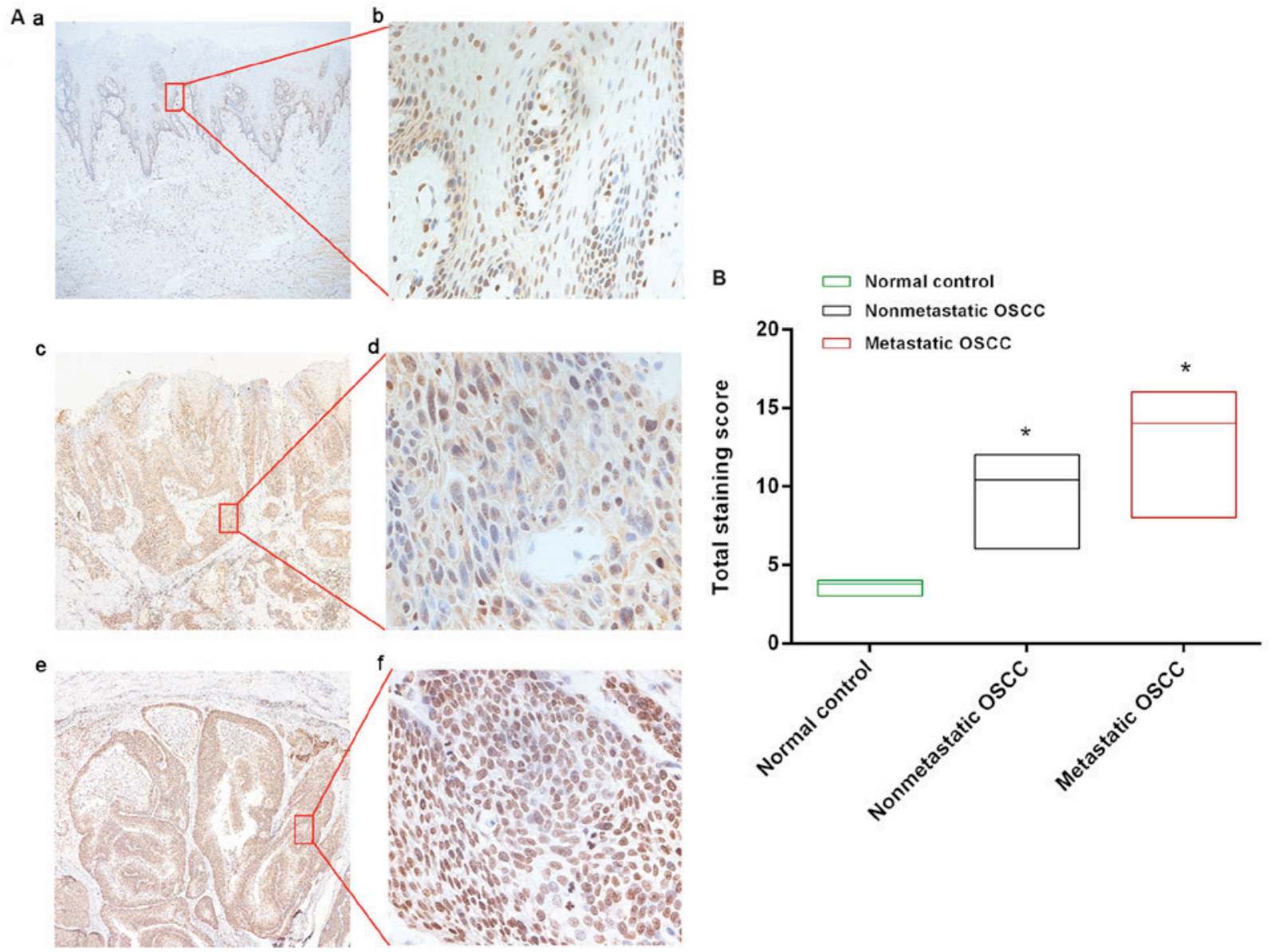

Figure 2. HMGN2 is expressed at a high level in human OSCC tissues. Immunohistochemistry results indicated positive staining for HMGN2 within the nucleus. Noncancerous control specimens demonstrated weakly positive reactivity, only in basal cells, whereas neoplastic cells of OSCC tissues demonstrated strongly positive HMGN2 expression, especially metastatic OSCC tissues. The images provided are representative of three independent experiments. (A) Normal human oral tissues at (a) $\mathrm{x} 40$ and (b) $\mathrm{x} 400$ magnification; non-metastatic OSCC tissues at (c) $\mathrm{x} 40$ and (d) $\mathrm{x} 400$ magnification; and, metastatic OSCC tissues at (e) $x 40$ and (f) $x 400$ magnification. (B) Floating bars were constructed from data obtained in three independent experiments. The total HMGN2 staining scores of non-metastatic and metastatic OSCC tissues were significantly increased compared with that of normal control tissue. Furthermore, the score of metastatic OSCC tissues was high compared with non-metastatic tissue. " $\mathrm{P}<0.05$ vs. normal control. HMGN2, high mobility group nucleosomal-binding domain 2; OSCC, oral squamous cell carcinoma.

staining, and $4(80 \%)$ metastatic OSCC tissues exhibited strong nuclear staining.

\section{Discussion}

HMG proteins are classified into super families on the basis of their physical and chemical properties. Each of these proteins contains a unique structural motif that induces specific changes in its binding sites and participates in distinct cellular functions (10). A total of $3 \mathrm{HMG}$ protein families are defined in terms of the structure of their DNA binding domains as well as their substrate binding specificity. The three families include the HMGA, HMGB and HMGN family $(11,12)$. These HMG proteins contribute to chromatin dynamics, the transcriptional activities of various genes and other cellular processes by distorting, bending or otherwise modifying the structure of DNA $(13,14)$.

The HMGN family comprises 5 chromatin architectural proteins that are present in higher vertebrates (13). Of these, HMGN1, 2 and 4 are expressed ubiquitously, whereas HMGN 3 and 5 are expressed in specific tissues $(4,15,16)$. The function of these proteins in transcription, DNA repair and cancer progression have been partly established $(3,4,17,18)$. The results of recent studies have demonstrated that HMGN1 may be a promising clinical biomarker for several types of cancer $(19,20)$. Similarly, the expression of HMGN5 (previously known as NSBP1) is highly regulated in various types of human cancer, including prostate, bladder, breast, lung and clear-cell renal-cell carcinoma (21). Knockdown of HMGN5 was demonstrated to suppress the viability and invasive ability of human urothelial bladder cancer cells (22), which further supports the involvement of HMGN5 in cancer progression.

HMGN2 is expressed ubiquitously in adult tissues and is markedly expressed during embryogenesis $(16,23)$. The HMGN2 gene is located on chromosome 1p36.1 and contains 6 exons (24). HMGN2 expression is downregulated during the physiological processes of myogenesis, erythrogenesis and chondrogenesis, as well as during embryonic organ formation, such as kidney development (10). Abnormal expression of the HMGN2 gene or protein has been associated with the development of neoplasms and autoimmune diseases (25-27). However, due to the poorly defined biological functions of this protein, further research is required. 
In the present study, HMGN2 expression was examined in 7 tumor cell lines. The results indicated that the expression levels of HMGN2 in the majority of tumor cell lines, particularly MDA-MB-468 and THP-1 cells, were increased compared with PDLCs (Fig. 1A and B). This result is in accordance with previous results that HMGN2 was increased in leukemia, breast cancer and small-intestine cancer cells, which suggested an effect of HMGN2 on transcription and cell differentiation in cancer progression (28). Based on the western blotting results in the present study, it is speculated that HMGN2 serves an important function in tumorigenesis (29). Oral cancer, comprising $>95 \%$ squamous cell carcinoma, is the sixth greatest public health concern globally in terms of increasing incidence and mortality rates $(30,31)$. For this reason, HMGN2 expression was examined inhuman OSCC tissues by immunohistochemistry. The expression of HMGN2 in OSCC tissues was increased compared with normal oral tissue. In addition, HMGN2 expression was increased in metastatic OSCC tissues compared with that in non-metastatic tissue. These results indicate that HMGN2 protein may be associated with tumor metastasis.

Oncogene and tumor-associated protein overexpression serves a key function in the progression of various forms of human cancer (32-34). The aim of the present study was to characterize the endogenous expression of HMGN2 in tumor cells. The results demonstrate that the expression of HMGN2 was significantly increased in the majority of tumor cell lines tested compared with control PDLCs. We hypothesized that HMGN2 may serve a vital function in the development and invasion of certain types of cancer, having previously examined the anti-tumor mechanism of HMGN2 protein on Tca8113 cells, an oral squamous cell carcinoma line in which the results demonstrated that HMGN2 could induce apoptosis in Tca8113 cells and S-phase cell cycle arrest in Tca8113 cells (35). Although additional in vitro and in vivo studies addressing HMGN2 during invasion and metastasis are required, the present study indicates that HMGN2 regulates the proliferation and metastasis of various types of human tumor cells in vitro. The main result of the present study, that HMGN2 suppression controlled the proliferation and metastasis of tumor cells, may have broad implications in tumor diagnosis and therapy.

\section{Acknowledgements}

The present study was supported by the National Natural Science Foundation of China (grant nos. 81372892, 81621062 and 81520108009), 111 Project of the Ministry of Education China (grant no. B14038), and the Research Foundation from the State Key Laboratory of Oral Disease Sichuan University (grant no. SKLOD201601).

\section{Competing interests}

The authors declare that they have no competing interests.

\section{References}

1. Grasser KD, Wurz A and Feix G: Isolation and characterization of high-mobility-group proteins from maize. Planta 185: 350-355,1991.

2. Bianchi ME and Agresti A: HMG proteins: Dynamic players in gene regulation and differentiation. Curr Opin Genet Dev 15: 496-506, 2005.
3. Gerlitz G: HMGNs, DNA repair and cancer. Biochim Biophys Acta 1799: 80-85, 2010.

4. Rochman M, Taher L, Kurahashi T, Cherukuri S, Uversky VN, Landsman D, Ovcharenko I and Bustin M: Effects of HMGN variants on the cellular transcription profile. Nucleic Acids Res 39: 4076-4087, 2011.

5. Shimahara H, Hirano T, Ohya K, Matsuta S, Seeram SS and Tate S: Nucleosome structural changes induced by binding of non-histone chromosomal proteins HMGN1 and HMGN2. FEBS Open Bio 3: 184-119, 12013.

6. Subramanian M, Gonzalez RW, Patil H, Ueda T, Lim JH, Kraemer KH, Bustin M and Bergel M: The nucleosome-binding protein HMGN2 modulates global genome repair. FEBS J 276: 6646-6657, 2009.

7. Vestner B, Bustin M and Gruss C: Stimulation of replication efficiency of a chromatin template by chromosomal protein HMG-17. J Biol Chem 273: 9409-9414, 1998.

8. Fiorillo AA, Medler TR, Feeney YB, Liu Y, Tommerdahl KL and Clevenger CV: HMGN2 inducibly binds a novel transactivation domain in nuclear PRLr to coordinate Stat5a-mediated transcription. Mol Endocrinol 25: 1550-1564, 2011.

9. Arnold LF and Baram P: In vitro culture of periodontal ligament cells. J Dent Res 51: 953-959, 1972.

10. Hock R, Furusawa T, Ueda T and Bustin M: HMG chromosomal proteins in development and disease. Trends Cell Biol 17: 72-79, 2007.

11. Bustin M: Revised nomenclature for high mobility group (HMG) chromosomal proteins. Trends Biochem Sci 26: 152-153, 2001.

12. Reeves R: Nuclear functions of the HMG proteins. Biochim Biophys Acta 1799: 3-14, 2010.

13. Gonzálezromero R, Eirínlópez JM and Ausió J: Evolution of high mobility group nucleosome-binding proteins and its implications for vertebrate chromatin specialization. Mol Biol Evol 32: 121-131, 2015.

14. Zhang Q and Wang Y: HMG modifications and nuclear function. Biochim Biophys Acta 28: 1799: 28-36, 2010.

15. Kugler JE, Deng T and Bustin M: The HMGN family of chromatin-binding proteins: Dynamic modulators of epigenetic processes. Biochim Biophys Acta 1819: 652-656, 2012.

16. Furusawa T and Cherukuri S: Developmental function of HMGN proteins. Biochim Biophys Acta 1799: 69, 2010.

17. Raymond R: High mobility group (HMG) proteins: Modulators of chromatin structure and DNA repair in mammalian cells. DNA Repair (Amst) 36: 122-136, 2015.

18. Liu BL and Wan Y: Research progression about the HMGN protein in tumor. J Int Oncol: 278-281, 2016.

19. Wei F, Yang F, Jiang X, Yu W and Ren X: High-mobility group nucleosome-binding protein 1 is a novel clinical biomarker in non-small cell lung cancer. Tumor Biol 36: 9405-9410, 2015.

20. Nie Y, Yang D and Oppenheim JJ: Alarmins and antitumor immunity. Clin Ther 38: 1042-1053, 2016.

21. Li DQ, Hou YF, Wu J, Chen Y, Lu JS, Di GH, Ou ZL, Shen ZZ, Ding J and Shao ZM: Gene expression profile analysis of an isogenic tumour metastasis model reveals a functional role for oncogene AF1Q in breast cancer metastasis. Eur J Cancer 42: 3274-3286, 2006.

22. Gan Y, Tan J, Yang J, Zhou Y, Dai Y, He L, Yao K and Tang Y: Erratum to: Knockdown of HMGN5 suppresses the viability and invasion of human urothelial bladder cancer 5,637 cells in vitro and in vivo. Med Oncol 32: 161, 2015.

23. Mohan G: Chromatin-binding HMGN proteins and the neuronal differentiation of enbryonal carcinoma cells in vitro. University of Glasgow, 2012.

24. Popescu N, Landsman D and Bustin M: Mapping the human gene coding for chromosomal protein HMG-17. Human Genet 85: 376-378, 1990.

25. Xie P, Deng LX, Gong P, Ding Y and Tang XH: Expression of HMGB1 and HMGN2 in gingival tissues, GCF and PICF of periodontitis patients and peri-implantitis. Braz J Microbiol 42: 1213-1219, 2011.

26. Deng LX, Wu GX, Cao Y, Fan B, Gao X, Tang XH and Huang N: The chromosomal protein HMGN2 mediates the LPS-induced expression of $\beta$-defensins in mice. Inflammation 35: 456-473, 2012.

27. Porkka K, Laakkonen P, Hoffman JA, Bernasconi M and Ruoslahti E: A Fragment of the HMGN2 protein homes to the nuclei of tumor cells and tumor endothelial cells in vivo. Proc Natl Acad Sci USA 99: 7444-7449, 2002.

28. Chengqian Y, Shuai S, Xue W, Yuting Z and Jianfa Y: Pathology Do: Research progress of high mobility group N2 in tumor. J Modern Oncol, 2016. 
29. Porkka K, Laakkonen P, Hoffman JA, Bernasconi $M$ and Ruoslahti E: A fragment of the HMGN2 protein homes to the nuclei of tumor cells and tumor endothelial cells in vivo. Proc Natl Acad Sci USA 99: 7444-7449, 2002.

30. Neville BW and Day TA: Oral cancer and precancerous lesions. CA Cancer J Clin 52: 195-215, 2002.

31. Choi S and Myers JN: Molecular pathogenesis of oral squamous cell carcinoma: Implications for therapy. J Dent Res 87: 14-32, 2008.

32. Andishehtadbir A, Najvani AD, Pardis S, Ashkavandi ZJ, Ashraf MJ, Khademi B and Kamali F: Metastasis-associated protein 1 expression in oral squamous cell carcinomas: Correlation with metastasis and Angiogenesis/Metastaz İlişkili protein 1 ekspresyonunun oral Skuamöz Hücreli karsinomlarda metastaz ve anjiyogenez ile İlişkisi. Turkish J Pathol 31: 9-15, 2015.
33. Li SH, Tian H, Yue WM, Li L, Li WJ, Chen ZT, Hu WS, Zhu YC and Qi L: Overexpression of metastasis-associated protein 1 is significantly correlated with tumor angiogenesis and poor survival in patients with early-stage non-small cell lung cancer. Ann Surg Oncol 18: 2048-2056, 2011.

34. Wu CC, Huang YS, Lee LY, Liang Y, Tang RP, Chang YS, Hsieh LL and Yu JS: Overexpression and elevated plasma level of tumor-associated antigen $90 \mathrm{~K} / \mathrm{Mac}-2$ binding protein in colorectal carcinoma. Proteomics Clin Appl 2: 1586-1595, 2008.

35. Hu A, Dong X, Liu X, Zhang P, Zhang Y, Su N, Chen Q and Feng Y: Nucleosome-binding protein HMGN2 exhibits antitumor activity in oral squamous cell carcinoma. Oncol Lett 7: $115-120,2014$. 